\title{
Metaanálisis del efecto de dietas bajas en proteína y adicionada con aminoácidos sintéticos para cerdos machos castrados en finalización
}

\author{
Meta-analysis of the effect of low protein diets for finishing barrows
}

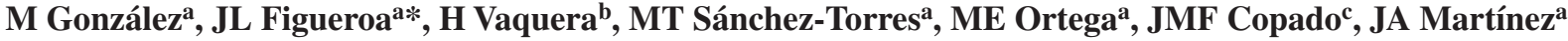 \\ aPrograma de Ganadería, Colegio de Postgraduados, Montecillo, Texcoco, México. \\ bPrograma de Estadística, Colegio de Postgraduados, Montecillo, Texcoco, México. \\ ${ }^{\mathrm{c}}$ Departamento de Zootecnia, Universidad Autónoma Chapingo, Texcoco, México.
}

\begin{abstract}
SUMMARY
A meta-analysis was carried out using individually pig data from seven experiments involving 222 hybrid finishing pigs with initial body weight of $52.69 \pm 3.74 \mathrm{~kg}$, individually housed, fed low-protein diets. Growth performance, carcass characteristics and plasma urea nitrogen concentration were evaluated. An analysis of variance and regression using a response surface model with RSREG procedure of SAS (2009) were performed considering level of crude protein (CP), effect of the experiment, year, time of year, type and level food additive supplemented. Optimum levels of CP were determined $(12.8,11.3$ and $9.5 \%)$ and corroborated through an additional experiment using 36 hybrid finishing pigs with $54.01 \mathrm{~kg}$ of initial weight, during 42 days. Average daily gain, daily feed intake, feed conversion, final weight, gain lean meat, lean meat percentage, longissimus muscle area and plasma urea nitrogen concentration were not affected $(\mathrm{P}>0.05)$ at a concentration of $11.5 \% \mathrm{CP}$, however, with $9.5 \% \mathrm{PC}$ these variables were affected $(\mathrm{P} \leq 0.05)$; backfat increased by lowering $\mathrm{CP}(\mathrm{P} \leq 0.05)$. In the experiment to corroborate optimal levels of $\mathrm{PC}$, there were no differences $(\mathrm{P}>0.05)$ for weight gain, feed intake, final weight and gain lean to lower the PC diet to $11.5 \%$. For backfat, longissimus muscle area and lean meat percentage there were no differences $(\mathrm{P}>0.05)$ when decreasing prot to $12.89 .5 \%$ PC. Plasma urea concentration was lowered $(\mathrm{P} \leq 0.05)$ to decrease the $\mathrm{PC}$ diet. Reducing dietary protein to $11.5 \%$ does not adversely affect the variables analysed.
\end{abstract}

Key words: Low-protein diets, meta-analysis, finishing barrows.

\section{RESUMEN}

Se realizó un metaanálisis con datos originales de siete experimentos con 222 cerdos híbridos en finalización alojados individualmente con peso vivo inicial promedio de 52,69 $\pm 3,74 \mathrm{~kg}$, alimentados con dietas bajas en proteína. Se evaluó el comportamiento productivo, las características de la canal y la concentración de urea en plasma. Se realizó análisis de varianza y de regresión utilizando un modelo de superficie de respuesta mediante RSREG de SAS (2009), considerando nivel de proteína cruda (PC) y efecto del experimento, año, época del año, tipo y nivel del aditivo alimenticio agregado. Se determinaron los niveles óptimos de PC (12,8, 11,3 y 9,5\%), corroborándolos con un experimento adicional utilizando 36 cerdos híbridos en finalización con 54,01 kg de peso inicial durante 42 días. La ganancia diaria de peso, consumo diario de alimento, conversión alimenticia, peso final, ganancia de carne magra, porcentaje de carne magra, área del músculo longissimus y concentración de urea en plasma, no se afectan $(\mathrm{P}>0,05)$ con $11,5 \%$ de $\mathrm{PC}$ pero al disminuir la PC hasta $9,5 \%$ sí son afectadas $(\mathrm{P} \leq 0,05)$; el grosor de la grasa dorsal aumenta cuando disminuye la $\mathrm{PC}(\mathrm{P} \leq 0,05)$. Al corroborar los niveles óptimos de PC no hubo diferencias en ganancia de peso, consumo de alimento, peso final y ganancia de carne magra $(\mathrm{P} \leq 0,05)$ al bajar la $\mathrm{PC}$ hasta 11,5\%. Para grasa dorsal, área del músculo longissimus y porcentaje de carne magra no hubo diferencias $(\mathrm{P}>0,05)$ al reducir la proteína de 12,8 hasta 9,5\%. La concentración de urea en plasma se redujo $(\mathrm{P} \leq 0,05)$ al disminuir la PC de la dieta. La reducción de proteína en la dieta hasta 11,5\% no afecta negativamente las variables analizadas.

Palabras clave: dietas bajas en proteína, metaanálisis, cerdos en finalización.

\section{INTRODUCCIÓN}

La reducción de la proteína cruda (PC) en la dieta (sorgo o maíz-pasta de soya), asociada con una adecuada adición de aminoácidos (AA) sintéticos, no afecta en la ganancia de peso y la eficiencia alimenticia de los cerdos y favorece a la reducción en la excreción de nitrógeno fecal y urinario (Osada et al 2011, Shriver et al 2003). La adecuada adición de AA sintéticos en dietas sorgo-pasta de soya, formuladas

Aceptado: 13.08.2015.

* jlfigueroa@colpos.mx con un menor contenido de proteína que el recomendado por el NRC (1998), no influye negativamente en el comportamiento productivo de los cerdos (Reyes et al 2012, Figueroa et al 2012). El uso de dietas bajas en proteína adicionada con AA sintéticos tienen efectos negativos en las características de la canal porque hay una menor ganancia de carne magra y una mayor acumulación de tejido adiposo (Yue et al 2008, Figueroa et al 2012). Sin embargo, cerdos en finalización alimentados con dietas bajas en proteína y baja concentración de energía no aumentan el grosor de la grasa dorsal e incluso incrementan el AML, pero la respuesta productiva no es la óptima (Figueroa et al 2004). Además, al bajar el contenido de 
proteína en la dieta adicionando AA se reduce la excreción de nitrógeno manteniendo el comportamiento productivo (Deng et al 2009, Le Bellego et al 2001); esta práctica es una alternativa de bajo costo para controlar olores y la emisión de amoniaco en la producción porcina intensiva (Feilberg et al 2010, Hayes et al 2004). El objetivo de esta investigación fue evaluar mediante metaanálisis el efecto de dietas bajas en proteína basadas en sorgo-pasta de soya para cerdos en finalización sobre la respuesta productiva, características de la canal y concentración de urea en plasma, y determinar, mediante ecuaciones de predicción, el nivel óptimo de PC en la dieta para la mejor respuesta de las variables mencionadas.

\section{MATERIAL Y MÉTODOS}

\section{BASE DE DATOS Y DISEÑO EXPERIMENTAL}

El conjunto de datos utilizados se derivó de una línea de investigación acerca de alimentación de cerdos con dietas bajas en proteína adicionadas con aminoácidos sintéticos bajo el concepto de cumplir la premisa de "proteína ideal" en la etapa de finalización. Los datos se encuentran publicados en revistas científicas y material no publicado (tesis de maestría) (cuadro 1). Se recopilaron de estudios realizados de 2005 a 2012. Los estudios analizados tenían en común evaluar diferentes niveles de proteína en dietas para cerdos en etapa de finalización. Los criterios que se utilizaron en este metaanálisis para el uso de los datos fueron, en primer lugar, que cada experimento debió tener más de una repetición de cada tratamiento, independiente del tamaño de la muestra. En segundo lugar tenía que haber una clara comparación de los resultados de los cerdos alimentados con una dieta con nivel de proteína estándar contra el desempeño de los cerdos alimentados con dietas que contenían nivel de proteína diferente al estándar. Y en tercer lugar el experimento tuvo que ser concurrente, es decir, que los tratamientos de cada experimento se aplicaran al mismo tiempo.

Fue importante que cada experimento contara con el conjunto de datos individuales de cada cerdo de cada experimento para asegurar que las omisiones no introdujeran sesgo y construir una base de datos suficientemente grande que fuera insensible a las variaciones en los valores de los distintos experimentos o de la adición o eliminación de los experimentos individuales. Se contó con datos de siete experimentos con 222 cerdos con un peso vivo inicial promedio de $52,69 \pm 3,74 \mathrm{~kg}$, siendo cada cerdo la unidad experimental (cuadro 1). En cada estudio los cerdos se alojaron en corral individual de $1,5 \times 1,2 \mathrm{~m}$, equipado con comedero tipo tolva de una boca y bebedero de chupón. En todos los experimentos el agua y el alimento se ofrecieron a libre acceso. La limpieza de corrales e inspección del estado de salud de los cerdos se realizó diariamente. El cambio de peso de los cerdos para calcular ganancia diaria de peso (GDP), consumo diario de alimento (CDA) y conversión alimenticia (CA) se obtuvo semanalmente. El último día de la fase experimental de cada trabajo (cuadro1) se tomaron muestras de sangre mediante punción en la vena cava utilizando un tubo Vacutainer ${ }^{\circledR}$ con heparina, que se colocaron en hielo hasta centrifugarse (centrifuga SIGMA 2-16k, Germany) durante 20 min a 2.500 g para separar el plasma y las células sanguíneas. El plasma se colocó en tubos de polipropileno y se congeló a $-20{ }^{\circ} \mathrm{C}$ (EUR251P7W Tappan, Electrolux Home Products North America, USA) hasta realizar las determinaciones de urea en plasma por espectrofotometría de absorción atómica (espectrofotómetro Cary 1E UV-vis, Varian, Australia; Chaney y Marbach 1962).

El día primero y último de la etapa en cada experimento se midió grasa dorsal (GD) y el área del músculo longissimus (AML) con un ultrasonido de tiempo real Sonovet 600 (Medison, Inc., Cypress, California, USA). Con estos datos y los de peso inicial y final se calculó la ganancia de

Cuadro 1. Resumen de los estudios utilizados para evaluar el comportamiento productivo, características de la canal y la concentración de urea en plasma en cerdos machos castrados en finalización.

Summary of the studies used to evaluate growth performance, carcass characteristics and plasma urea concentration in finishing barrows.

\begin{tabular}{|c|c|c|c|c|c|c|c|}
\hline Estudio & $\begin{array}{c}\text { Tipo de } \\
\text { publicación }\end{array}$ & $\begin{array}{l}\mathrm{N}^{\mathrm{o}} \mathrm{de} \\
\text { cerdos }\end{array}$ & Raza & Sexo & PV inicial & PV final & $\begin{array}{l}\text { Periodo } \\
\text { días }\end{array}$ \\
\hline $\begin{array}{l}\text { Martínez- Aispuro } \\
\text { (2005) }\end{array}$ & $\begin{array}{l}\text { Tesis de } \\
\text { maestría }\end{array}$ & 24 & Landrace $\times$ Yorkshire $\times$ Duroc & Machos castrados & 48,6 & 74,0 & 28 \\
\hline Reyna et al (2006) & Artículo & 32 & Yorkshire $\times$ Duroc $\times$ Pietrain & Machos castrados & 50,3 & 91,8 & 42 \\
\hline Zamora et al (2010) & Artículo & 32 & Yorkshire $\times$ Duroc $\times$ Pietrain & Machos castrados & 49,5 & 82,4 & 35 \\
\hline Rivera et al (2010) & Artículo & 40 & Yorkshire $\times$ Duroc $\times$ Pietrain & Machos castrados & 56,5 & 87,4 & 35 \\
\hline Rivera et al (2010) & Artículo & 36 & Yorkshire $\times$ Duroc $\times$ Pietrain & Machos castrados & 58,4 & 92,9 & 35 \\
\hline Reyes et al (2012) & Artículo & 36 & Landrace $\times$ Yorkshire $\times$ Duroc & Machos castrados & 51,3 & 81,9 & 35 \\
\hline Figueroa et al (2012) & Artículo & 36 & YorkshirexDuroc $\times$ Pietrain & Machos castrados & 54,3 & 99,8 & 56 \\
\hline
\end{tabular}

Las dietas utilizadas en todos los experimentos fueron a base de sorgo-pasta de soya.

En todos los experimentos las variables respuesta fueron: GDP, CDA, CA, PF, GCM, GD, AML, PCM y urea en plasma. 
carne magra (GCM) y el porcentaje de carne magra (PCM) en la canal, con la ecuación del NPPC (1991).

\section{ANÁLISIS ESTADÍSTICO}

Para investigar el efecto de los factores sobre las variables se utilizó el siguiente modelo: $\mathrm{Y}_{\mathrm{ijk}}=\mu+\mathrm{T}_{\mathrm{i}}+\gamma_{\mathrm{j}}$ $+\alpha_{\mathrm{k}}+b_{1} \mathrm{X}_{1 \mathrm{ijk}}+b_{2} \mathrm{X}_{2 \mathrm{ijk}}+b_{3} \mathrm{X}_{3 \mathrm{ijk}}+\varepsilon_{\mathrm{ijk}}$; donde $\mu=$ media general; $\mathrm{T}_{\mathrm{i}}=$ efecto del i-ésimo experimento; $\gamma_{\mathrm{j}}=$ efecto de la j-ésima época del año; $\alpha_{\mathrm{k}}=$ efecto del k-ésimo nivel de proteína cruda, $=\mathrm{X}_{1 \mathrm{ijk} 1}=$ peso inicial $\mathrm{i}$-ésimo experimento, $X_{2 i j k}=$ ganancia diaria de peso en la j-ésima época del año, $X_{3 \mathrm{ijk}}=$ consumo diario de alimento en el k-ésimo nivel de proteína; $b_{1}, b_{2}, b_{3}=$ parámetros de las covariables de medida; $\varepsilon_{\mathrm{ijk}}=$ error experimental asociado con cada medición. Del análisis de varianza se obtuvieron los promedios del comportamiento productivo, dentro de las cuales se consideró CDA, GDP, CA, GCM y PF; y en las características de la canal se contempló: GD, PCM, AML; y la concentración de urea en plasma.

Posteriormente se realizó un análisis de regresión lineal y cuadrática usando un modelo de superficie de respuesta con el comando RSREG de SAS (2009): técnica de optimización basada en planeamientos factoriales para obtener el nivel de proteína para las diferentes variables productivas, características de la canal y concentración de urea en plasma. El modelo de regresión de superficie de respuesta que se utilizó fue: $Y_{i}=\beta_{0}+\beta_{1} X_{i}+\beta_{2} X_{i}^{2}+$ $\varepsilon_{\mathrm{i}}$; donde $\mathrm{Y}_{\mathrm{i}}=$ variable dependiente, $\beta_{0}$ a $\beta_{2}=$ coeficientes estimados, $\mathrm{X}_{\mathrm{i}}=$ nivel de $\mathrm{PC}$.

\section{EXPERIMENTO PARA CORROBORAR EL METAANÁLISIS}

Una vez que se analizaron los datos de los experimentos con el metaanálisis se prosiguió a montar un experimento para corroborar los resultados de las regresiones, de donde se obtuvo el nivel de proteína cruda óptimo para cada variable productiva, características de la canal y concentración de urea en plasma. Los niveles de proteína utilizados en el experimento de corroboración fueron los de las variables que presentaron mayores valores de $\mathrm{R}^{2}$ en el modelo de regresión a GDP y CDA (12,8 y 11,3\%). Adicionalmente se formuló una tercera dieta con 9,5\% de PC, valor que consistentemente había producido buenos resultados productivos en los experimentos utilizados en el metaanálisis.

El experimento se efectuó en la Unidad Porcina de la Granja Experimental del Colegio de Postgraduados, en el Campus Montecillo, Texcoco, estado de México, localizada a $98^{\circ} 48^{\prime} 27^{\prime \prime}$ O y a $19^{\circ} 48^{\prime} 23^{\prime \prime}$ N y una altitud de $2.241 \mathrm{~m}$, con clima templado subhúmedo con lluvias en verano, temperatura media anual de $15,2^{\circ} \mathrm{C}$ y precipitación media anual de 644,8 mm (García 1988). Se utilizaron 18 cerdos machos castrados y 18 hembras (Yorkshire $\times$ Duroc $\times$ Pietrain) en la etapa de finalización durante 42 días, con un peso promedio de $54,01 \mathrm{~kg}$; el diseño experimental fue completamente al azar, para evaluar tres concentraciones de PC, con doce repeticiones por tratamiento.

Las dietas (cuadro 3) se formularon basadas en sorgopasta de soya adicionadas con L- Lisina. $\mathrm{HCl}$, L-treonina, DL-metionina y L-triptófano sintéticos, agregados hasta alcanzar los requerimientos nutricionales de aminoácidos digestibles para la etapa de finalización (NRC 1998), bajo la premisa de proteína ideal. Los cerdos recibieron las mismas condiciones de alojamiento y manejo que en los experimentos utilizados en el metaanálisis, y las variables analizadas (GDP, CDA, CA, PF, GCM, GD, AML, PCM $\mathrm{y}$ urea en plasma) se midieron de igual manera.

\section{RESULTADOS}

\section{METAANÁLISIS}

Los resultados del metaanálisis para las variables productivas (figura 1) mostraron que dietas con baja proteína en la etapa de finalización, para GDP, disminuyó la respuesta al bajar el nivel de proteína en la dieta de 14,1 a 9,5\% (0,954 $\mathrm{kg} \mathrm{d}^{-1}$ a 0,824 $\left.\mathrm{kg} \mathrm{d}^{-1} \mathrm{P} \leq 0,05\right)$. Para CDA, el mayor consumo $(\mathrm{P} \leq 0,05)$ se encontró con $14 \%(3,191$ $\mathrm{kg} \mathrm{d}^{-1}$ ) y el menor utilizando $10,1 \%\left(2,613 \mathrm{~kg} \mathrm{~d}^{-1}\right)$ de PC; mientras que $\mathrm{CA}$ fue mejor $(\mathrm{P} \leq 0,05)$ cuando el nivel fue $12,5 \%$ en comparación con $9,5 \%$ (2,811 vs. 3,35$)$, pero $10,1,14$ y 14,1\% PC fueron similares (P > 0,05). En el PF (figura 3) se observó que al utilizar 10,1, 11,8 y 14\% de $\mathrm{PC}$ en la dieta hay diferencias $(\mathrm{P} \leq 0,05 ; 84,41,100,32 \mathrm{y}$ $88,59 \mathrm{~kg}$ ) entre ellos; pero con $9,5,12,1$ y $14,1 \%$ no hubo diferencias ( $\mathrm{P}>0,05 ; 86,02,86,88$ y $85,89 \mathrm{~kg}$ ). Para GCM no hubo diferencias $(\mathrm{P}>0,05)$ cuando se utilizaron 9,5, 12,5 y $14 \%$ PC $\left(0,37,0,39\right.$ y $\left.0,37 \mathrm{~kg} \mathrm{~d}^{-1}\right)$, pero sí $(\mathrm{P} \leq 0,05)$ cuando se disminuyó de 12,5 a $11,8 \%$ PC (0,39 a 0,31 $\left.\mathrm{kg} \mathrm{d}^{-1}\right)$. Para GD se encontraron diferencias $(\mathrm{P} \leq 0,005)$ cuando se utilizaron diferentes niveles de 9,5 a $14,1 \%$ PC (figura 2). En el caso de PCM (figura 3), cuando se incluyó $11,8 \%$ PC se observó mayor $(38,48 \%)$ porcentaje $(\mathrm{P} \leq 0,05)$, pero los valores fueron similares $(\mathrm{P}>0,05)$ con $9,5,10,1,12,1$ y $14,1 \%(37,73,37,52,37,45$ y $37,41 \%)$. En el AML, los valores fueron significativamente diferentes $(\mathrm{P} \leq 0,05)$ con $12,1,12,5$ y $14 \%\left(29,84,23,92\right.$ y $\left.28,23 \mathrm{~cm}^{2}\right)$ pero similares con 9,5, 10,1 y 11,8\% $\mathrm{PC}(\mathrm{P}>0,05 ; 26,43$, 26,03 y $\left.26,97 \mathrm{~cm}^{2}\right)$. La concentración de urea en plasma (figura 4) disminuyó $(\mathrm{P} \leq 0,05)$ de 15,72 a 2,51 $\mathrm{mg} \mathrm{dL}^{-1}$, al reducir la PC de la dieta de 12,5 a 9,5\%.

\section{ECUACIONES DE PREDICCIÓN}

Los valores de $\mathrm{R}^{2}$ de las ecuaciones de predicción obtenidas mediante el modelo de regresión cuadrática indican que la ecuación muestra un ajuste aceptable para modelar la respuesta productiva, características de la canal y concentración de urea en plasma. Las variables respuesta que presentaron los mayores valores de $\mathrm{R}^{2}$ fueron urea, GDP, CDA y AML, con 12,7, 12,8, 11,3 y 11,4\% de PC; 
GDP kg d $^{-1} \quad \square$ CDA kg d $^{-1} \quad \square \mathrm{CA}$

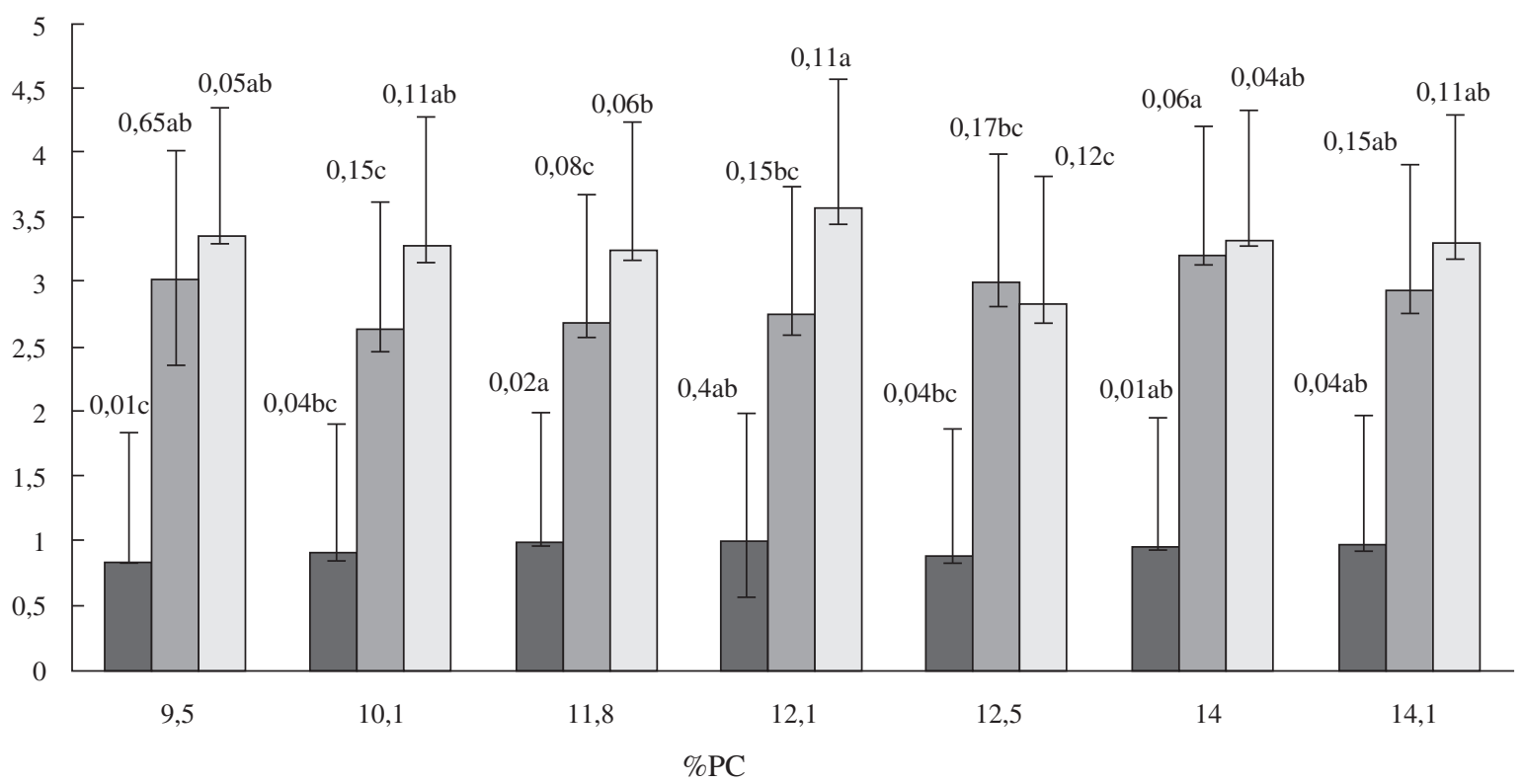

Figura 1. Metaanálisis del efecto del nivel de PC en dietas para cerdos machos castrados en finalización sobre las variables ganancia diaria de peso (GDP), consumo diario de alimento (CDA) y conversión alimenticia (CA). feed:gain ratio.

Meta-analysis of the effect of the crude protein level in diets for finishing barrows on average daily gain, average daily feed intake and

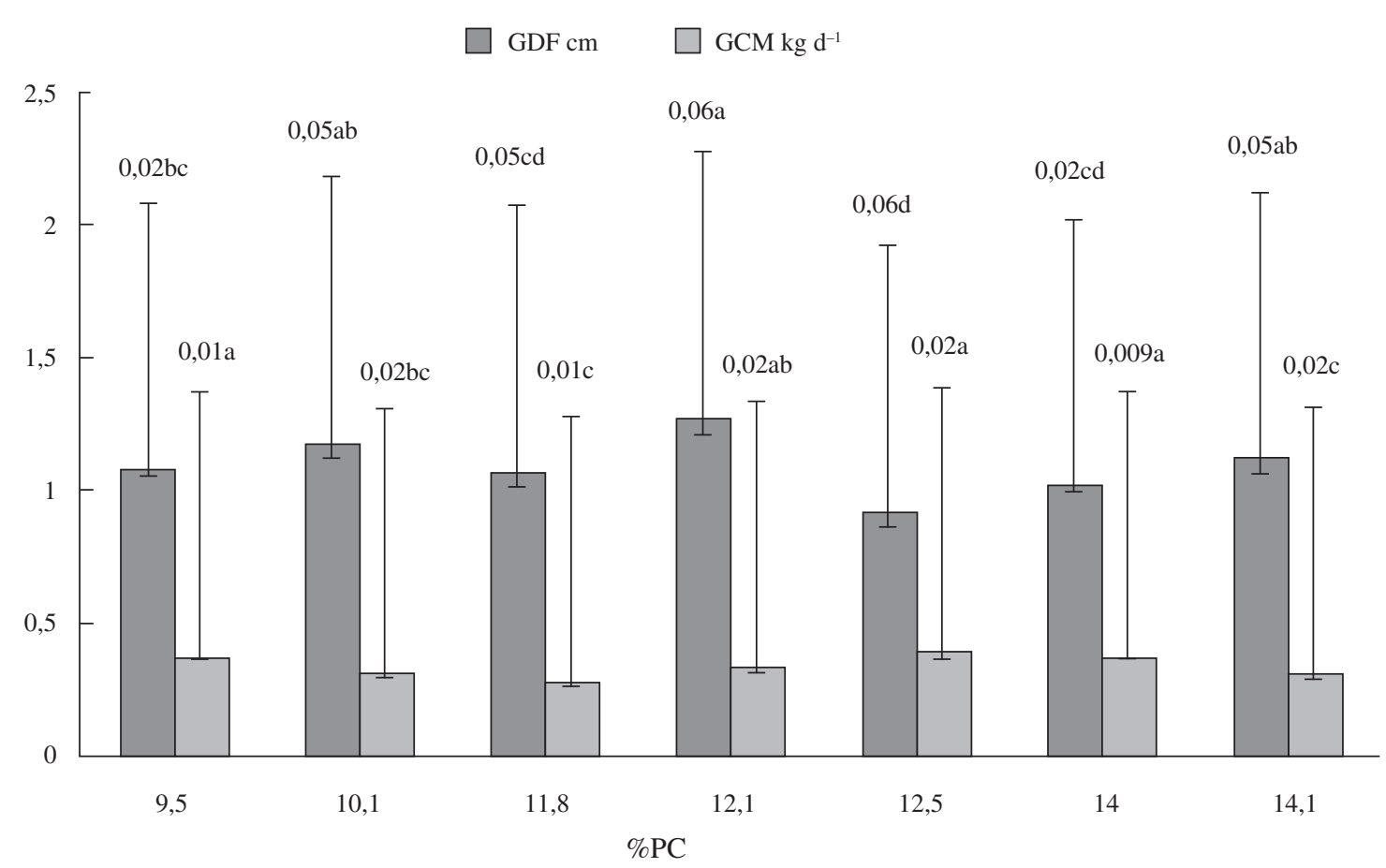

Figura 2. Metaanálisis del efecto del nivel de PC en dietas para cerdos machos castrados en finalización sobre las variables grasa dorsal final (GDF) y ganancia de carne magra (GCM).

Meta-analysis of the effect of crude protein level in diets for finishing barrows on the backfat thickness and the fat free lean gain. 


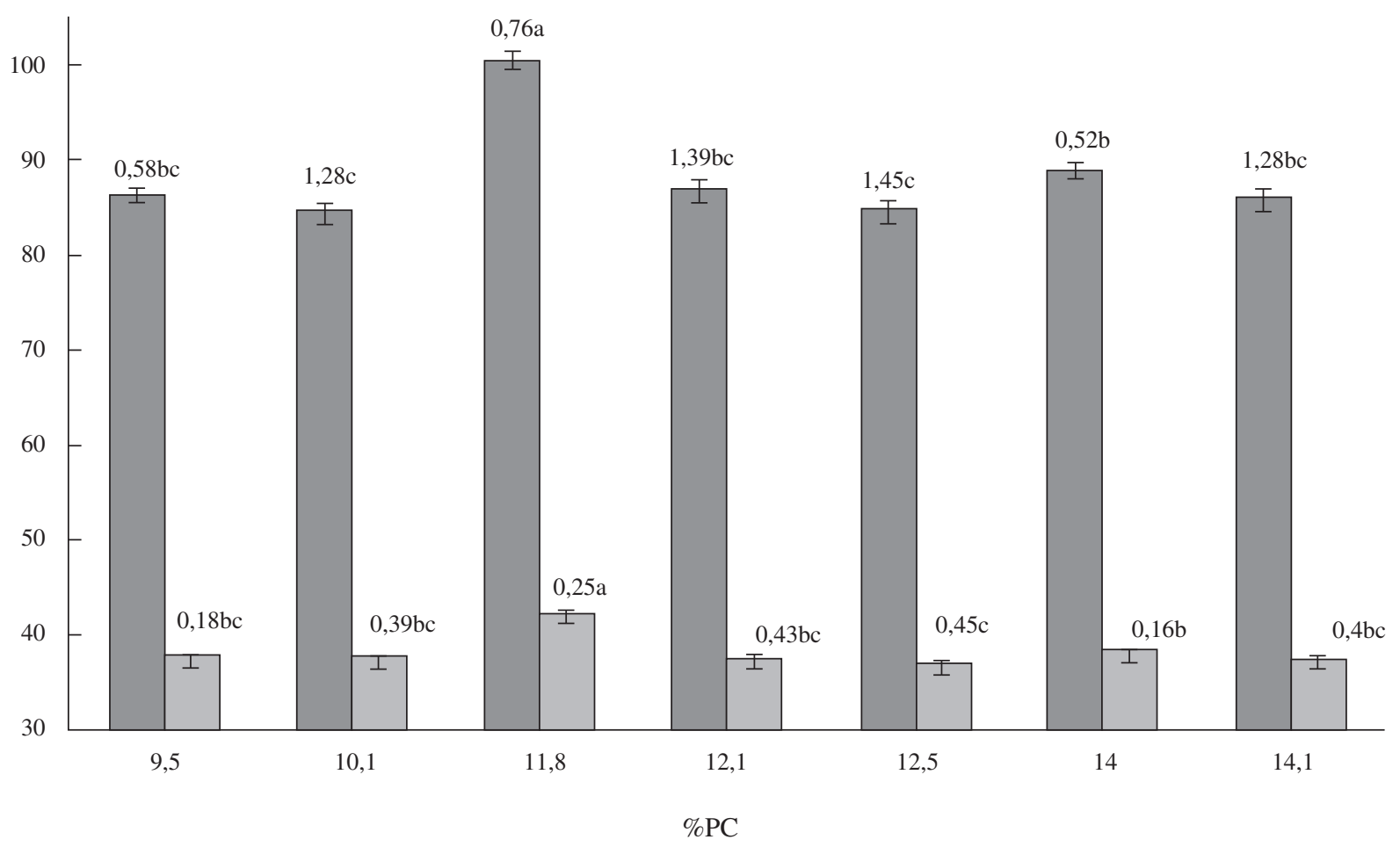

Figura 3. Metaanálisis del efecto del nivel de PC en la dieta de cerdos machos castrados en finalización sobre las variables porcentaje de carne magra final (\% CMF) y peso final $(\mathrm{PF})$.

Meta-analysis of the effect of crude protein level in the diet of finishing barrows on lean meat percentage and final body weight.

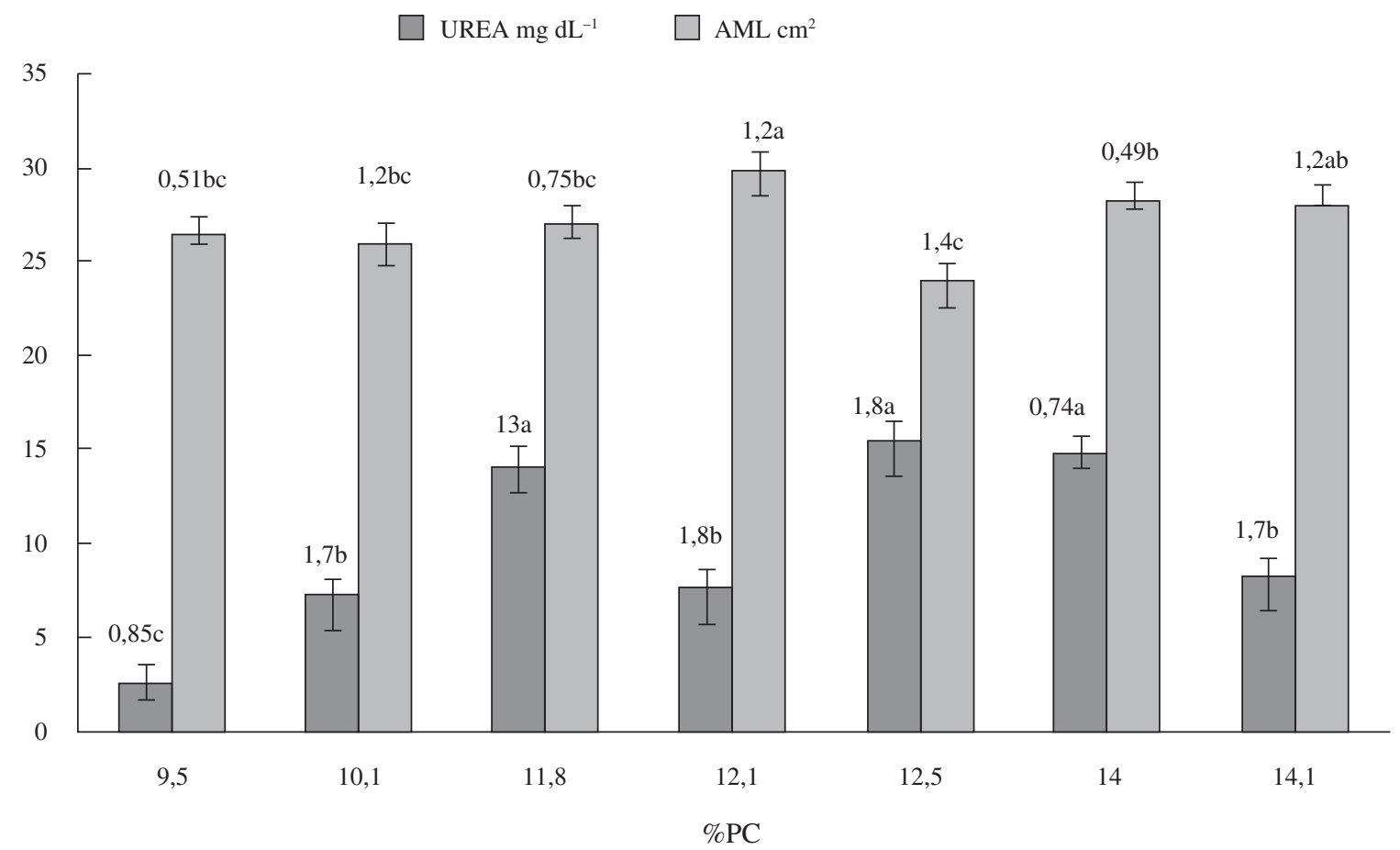

Figura 4. Metaanálisis del efecto del nivel de PC en la dieta de cerdos machos castrados en finalización sobre las variables área del músculo longissimus (AML) y concentración de urea en plasma (UREA).

Meta-analysis of the effect of crude protein level in the diet of finishing barrows on variables longissimus muscle area and plasma urea nitrogen concentration. 
Cuadro 2. Nivel de proteína para obtener la mayor respuesta en la ganancia diaria de peso, consumo de alimento, concentración de urea en plasma y área del músculo longissimus de cerdos machos castrados en finalización.

Protein level to get the highest response in average daily gain, average daily feed intake, plasma urea nitrogen concentration and longissimus muscle area of finishing barrows.

\begin{tabular}{ccccc}
\hline Variable & $\begin{array}{c}\text { Nivel PC } \\
(\%)\end{array}$ & Ecuación de predicción & $\mathrm{R}^{2}$ & $\mathrm{P}$ \\
\hline GDP & 12,8 & $-0,73387+0,2626(\mathrm{PC})-0,01095\left(\mathrm{PC}^{2}\right)$ & 0,539 & 0,021 \\
CDA & 11,3 & $8,4677-1,01643(\mathrm{PC})+0,04502\left(\mathrm{PC}^{2}\right)$ & 0,469 & 0,028 \\
UREA & 12,7 & $-141,31+24,2108(\mathrm{PC})-0,94967\left(\mathrm{PC}^{2}\right)$ & 0,617 & 0,015 \\
AML & 11,4 & $77,681-9,304(\mathrm{PC})+0,4085\left(\mathrm{PC}^{2}\right)$ & 0,432 & 0,016 \\
\hline
\end{tabular}

$\mathrm{NPC}=$ nivel de proteína cruda; $\mathrm{GDP}=$ ganancia diaria de peso; $\mathrm{CDA}=$ consumo diario de alimento; $\mathrm{UREA}=$ concentración de urea en plasma, $\mathrm{AML}=$ área del musculo longuissimus. $\mathrm{R}^{2}=$ coeficiente de determinación, $\mathrm{P}=$ nivel de significancia.

en cuadro 2 se muestran los valores de $\mathrm{R}^{2}$ y los óptimos de proteína cruda.

\section{RESPUESTA A LOS NIVELES ÓPTIMOS DE PC OBTENIDOS DEL METAANÁLISIS}

Los machos castrados y las hembras no mostraron diferencias significativas $(\mathrm{P}>0,05)$ para las variables estudiadas, por lo que se procedió a realizar el análisis estadístico de manera global.

Se observaron diferencias (cuadro 4) para las variables productivas $\mathrm{PF}$, GDP y CDA $(\mathrm{P} \leq 0,05)$ cuando disminuye de 11,3 a 9,5\% la proteína cruda en la dieta, excepto para $\mathrm{CA}$, donde no hubo diferencias $(\mathrm{P}>0,05)$. Los cerdos alimentados con $12,8,11,3$ y 9,5\% PC mostraron diferencia $(\mathrm{P} \leq 0,05)$ para $\operatorname{GCM}\left(254,298\right.$ y $\left.212 \mathrm{~g} \mathrm{~d}^{-1}\right)$. Para las características de la canal, las variables GD, AML y PCM no presentaron diferencias $(\mathrm{P}>0,05)$ entre tratamientos. La concentración de urea en plasma disminuyó $(\mathrm{P} \leq 0,05)$ en $32,5 \%$ cuando el nivel de PC en la dieta se redujo de $12,8 \%$ a $9,5 \%$ (cuadro 4 ).

\section{DISCUSIÓN}

\section{COMPORTAMIENTO PRODUCTIVO}

Cuando las dietas bajas en proteínas están equilibradas en términos de AA esenciales es factible reducir los niveles de CP sin afectar el crecimiento de tejido magro, o ganancia de peso corporal. En la etapa de finalización los resultados del metaanálisis mostraron que al reducir la proteína cruda en 4,6 unidades porcentuales en dietas a base de sorgo-pasta de soya afectó negativamente todas las variables productivas (GDP, CDA, CA, PF y GCM), lo que podría deberse a la deficiencia de algunos AA esenciales (cuadro 3) en las dietas bajas en PC; esto también lo encontraron Ward y Southern (1995) en dietas con baja PC para cerdos en finalización donde disminuyó el contenido de los AA esenciales histidina, isoleucina y valina, respecto de los requerimientos (Figueroa et al 2004).
También, la menor respuesta de DBP puede atribuirse a que con la reducción en el contenido de proteína en la dieta puede faltar nitrógeno necesario para la síntesis de AA no esenciales (Tuitoek et al 1997, Heger et al 1998), ya que en condiciones de adecuado uso de la proteína parte de los AA esenciales son parcialmente degradados y usados en la síntesis de AA no esenciales (Heger et al 1998). Esto difiere de lo reportado por otros autores donde no se presentaron diferencias en las variables productivas cuando se redujo el contenido de PC más AA sintéticos en dietas a base de sorgo-pasta de soya (Figueroa et al 2012, Martínez-Aispuro et al 2012, Reyes et al 2012).

\section{CARACTERÍSTICAS DE LA CANAL}

El metaanálisis mostró que para cerdos machos castrados en finalización la disminución de la PC en la dieta afectó negativamente las características de la canal (GD, PCM, AML), lo que fue similar a lo que reportaron Figueroa $e t$ al (2012), donde aumentó la GD, disminuyó el PCM y el AML cuando se redujo en dos puntos porcentuales la PC y se adicionó lisina. La disminución en la ganancia de tejido magro y el aumento en el grosor de la GD de cerdos con las DBP se atribuye a la mayor disponibilidad de energía para la síntesis de lípidos y acumulación de tejido adiposo, como resultado de la reducción de energía para eliminar el exceso de proteína en la dieta (Figueroa et al 2002, Gómez et al 2002). Esto difiere de los resultados obtenidos por otros investigadores que al disminuir la PC en la dieta no observaron efecto sobre las características de la canal (Figueroa et al 2004, Zamora et al 2010, Martínez-Aispuro et al 2012, Reyes et al 2012). También se aumentan la retención de energía metabolizable en tejidos corporales, principalmente como grasa por tanto la canal del cerdo presenta mayor espesor de la grasa dorsal (Le Bellego et al 2001).

\section{CONCENTRACIÓN DE UREA EN PLASMA}

La urea excretada en la orina es el principal producto final nitrogenado del catabolismo AA en cerdos, y la 
Cuadro 3. Composición de las dietas para cerdos machos castrados en finalización del experimento de corroboración de niveles óptimos de baja proteína.

Composition of diets for finishing barrows in the experiment to corroborate the optimal low-protein levels.

\begin{tabular}{|c|c|c|c|}
\hline Tratamiento & $\mathrm{T} 1$ & $\mathrm{~T} 2$ & $\mathrm{~T} 3$ \\
\hline \multicolumn{4}{|l|}{ Ingrediente, $\%$} \\
\hline Sorgo & 82,979 & 87,05 & 91,94 \\
\hline Soya & 11,934 & 7,55 & 2,30 \\
\hline Aceite crudo de soya & 2,086 & 2,13 & 2,19 \\
\hline Ortofosfatos & 0,684 & 0,71 & 0,74 \\
\hline $\mathrm{CaCO}_{3}$ & 1,332 & 1,34 & 1,34 \\
\hline Sal común & 0,350 & 0,35 & 0,35 \\
\hline Vitaminas $* *$ & 0,150 & 0,15 & 0,15 \\
\hline Minerales $* * *$ & 0,100 & 0,10 & 0,10 \\
\hline Tripto + Plus $* * * *$ & 0,312 & 0,45 & 0,62 \\
\hline DL-Metionina & 0,0136 & 0,05 & 0,09 \\
\hline L-Treonina & 0,0602 & 0,12 & 0,18 \\
\hline \multicolumn{4}{|l|}{ Análisis calculado } \\
\hline EM, Mcal Kg-1 & 3,26 & 3,26 & 3,26 \\
\hline Proteína cruda, \% & 12,80 & 11,30 & 9,50 \\
\hline $\mathrm{Ca}$ & 0,70 & 0,70 & 0,70 \\
\hline Fósforo total & 0,44 & 0,43 & 0,42 \\
\hline Fósforo disponible & 0,23 & 0,23 & 0,23 \\
\hline Lisina, $\%$ & 0,66 & 0,66 & 0,66 \\
\hline Treonina, $\%$ & 0,43 & 0,43 & 0,43 \\
\hline Triptófano, \% & 0,17 & 0,17 & 0,17 \\
\hline Metionina + cistina, $\%$ & 0,39 & 0,39 & 0,39 \\
\hline Arginina, $\%$ & 0,62 & 0,50 & 0,35 \\
\hline Histidina, \% & 0,28 & 0,24 & 0,20 \\
\hline Isoleucina, \% & 0,53 & 0,46 & 0,38 \\
\hline Leucina, $\%$ & 1,34 & 1,26 & 1,16 \\
\hline Valina, $\%$ & 0,60 & 0,53 & 0,46 \\
\hline Fenilalanina + tirosina & 1,08 & 0,97 & 0,83 \\
\hline \multicolumn{4}{|l|}{ Análisis determinado } \\
\hline Proteína cruda, \% & 15,13 & 14,48 & 10,24 \\
\hline Calcio, $\%$ & 1,02 & 0,68 & 0,90 \\
\hline Fósforo total, \% & 0,94 & 0,49 & 0,52 \\
\hline
\end{tabular}

Sugerencia de nutrimentos para cerdos en la etapa de crecimiento (20-50 kg de peso vivo) por el NRC (1988).

** Cada kg de alimento aportó: Vit. A, 15,000 UI; Vit. D, 2,500 UI; Vit. E, $37.5 \mathrm{UI}$; Vit. K, $2.5 \mathrm{mg}$; tiamina, $2.25 \mathrm{mg}$; riboflavina, 6,25 $\mathrm{mg}$; niacina, $50 \mathrm{mg}$; cianocobalamina, $0,0375 \mathrm{mg}$; biotina, 0.13 mg; ácido fólico, $1,25 \mathrm{mg}$; ácido pantoténico, $20 \mathrm{mg}$; colina, 563 $\mathrm{mg}$; ***Aportó por cada kg de alimento: $\mathrm{Fe}, 150 \mathrm{mg} ; \mathrm{Zn}, 150 \mathrm{mg}$; Mn 150 mg; Cu, 10 mg; Se, 0.15 mg; I, 0,9 mg; Cr, 0,2 mg.

**** Tripto-Plus contiene: proteína cruda, $95 \%$; lisina, 55,3\%; treonina, $0,15 \%$; triptófano, $15 \%$; valina, $0,5 \%$; metionina+cistina, $1,75 \%$.

concentración de urea plasmática puede ser indicativo de $\mathrm{N}$ excretado en la orina (Zervas y Zijlstra 2002). Al bajar el contenido de proteína en la dieta adicionando AA sintéticos se reduce la excreción de N, manteniendo el comportamiento productivo (Le Bellego et al 2001). Una forma indirecta de medir esta reducción es por medio de la concentración de urea en plasma, variable que se relaciona linealmente y en forma positiva con la excreción urinaria de $\mathrm{N}$ en cerdos alimentados ad libitum (Zervas y Zijlstra 2002). La concentración de urea plasmática es $36 \%$ menor en cerdos en finalización (Kendall et al 1998, Le Bellego et al 2002); se ha observado que la reducción es proporcional a la disminución de la proteína dietaria (Figueroa et al 2002).

La concentración de urea en plasma disminuyó cuando se redujo el nivel de PC en la dieta, observándose una reducción considerable cuando bajó de 12,5 a 9,5\% PC (15,72 a 2,51 $\left.\mathrm{mg} \mathrm{dL}^{-1}\right)$. Esto coincide con los datos obtenidos por otros investigadores (Figueroa et al 2002, 2003, Gómez et al 2002, Kerr et al 2003, Nyachoti et al 2006, Zamora et al 2010, Martínez-Aispuro et al 2009, Martínez-Aispuro et al 2012, Reyes et al 2012).

\section{RESPUESTA A LOS NIVELES ÓPTIMOS DE PC OBTENIDOS}

Los resultados obtenidos utilizando los niveles óptimos de PC muestran que para cerdos en finalización utilizar niveles de 12,8 y 11,3\% PC utilizando sorgo-pasta de soya y adicionada con AA sintéticos no se afectan las variables productivas, pero la reducción de la proteína en la dieta hasta $9,5 \%$ afecta negativamente la respuesta productiva, excepto para CA (Kerr et al 2004). Estos resultados son similares a los reportados por Reyes et al (2012) y MartínezAispuro et al (2012), pero esto difiere con lo reportado por Figueroa et al (2004, 2012), donde al disminuir la PC se afectó negativamente la respuesta productiva en cerdos en finalización. Para las características de la canal, disminuir la PC de 12,8 a 9,5 no tuvo ningún efecto negativo. Esto es similar a lo obtenido por otros investigadores, que al bajar la PC en la dieta no tuvo efecto sobre las características de la canal (Figueroa et al 2012, Martínez-Aispuro et al 2012, Reyes et al 2012). Al disminuir la proteína en la dieta los niveles de urea en plasma se reducen linealmente (Figueroa et al 2002, 2003, Gómez et al 2002, Kerr et al 2003), como se observó en el presente experimento, que al disminuir de 12,8 a 9,5\% la PC se redujo de 14,465 a $9,7676 \mathrm{mg} \mathrm{dL}^{-1}$ la urea.

Es posible concluir que, de acuerdo con lo observado en el metaanálisis, reducir de 14 a 9,5\% el nivel de PC en la dieta para cerdos en etapa de finalización alimentados con dietas sorgo-pasta de soya adicionadas con AA sintéticos, afecta negativamente todas las variables productivas, características de la canal y una disminución en la concentración de urea en plasma.

El nivel óptimo de PC (12,8 a 11,3\%) sugiere que los cerdos en la etapa de finalización se pueden alimentar con dietas bajas en proteína bruta $(11,3 \%)$ basadas en sorgoharina de soja adicionadas con AA sintéticos sin afectar las variables productivas, ni características de la canal y disminuyendo la concentración de urea en plasma. 
Cuadro 4. Efecto del nivel óptimo de proteína cruda en la dieta sobre el comportamiento productivo, características de la canal y concentración de urea en plasma de cerdos machos castrados en finalización. of finishing barrows.

Effect of optimal level of crude protein in the diet on growth performance, carcass characteristics and plasma urea nitrogen concentration

\begin{tabular}{|c|c|c|c|c|c|c|c|c|c|c|c|c|}
\hline & $\mathrm{PC}$ & EM & PI & $\mathrm{PF}$ & GDP & $\mathrm{CDA}$ & $\mathrm{CA}$ & GCM & GD & AML & PCM & U \\
\hline 1 & 12,8 & 3,265 & 53,59 & $86,71^{\mathrm{ab}}$ & $0,78^{a}$ & $2,64^{\mathrm{ab}}$ & 3,60 & $0,25^{\mathrm{b}}$ & 11,64 & 29,32 & 38,51 & $14,46^{\mathrm{a}}$ \\
\hline 2 & 11,3 & 3,265 & 54,93 & $90,27^{\mathrm{a}}$ & $0,86^{\mathrm{a}}$ & $2,82^{\mathrm{a}}$ & 3,20 & $0,30^{\mathrm{a}}$ & 11,29 & 31,47 & 39,01 & $12,38^{\mathrm{ab}}$ \\
\hline 3 & 9,5 & 3,265 & 53,38 & $82,42^{b}$ & $0,68^{\mathrm{b}}$ & $2,44^{\mathrm{b}}$ & 3,60 & $0,21^{\mathrm{c}}$ & 10,42 & 27,81 & 38,86 & $9,77^{\mathrm{b}}$ \\
\hline $\mathrm{EE}$ & & & & 1,56 & 0,37 & 0,10 & 0,21 & 0,01 & 0,46 & 1,50 & 0,46 & 0,92 \\
\hline \multicolumn{13}{|c|}{ Fuente de variación Valor de P } \\
\hline $\mathrm{PC}$ & & & & 0,006 & 0,006 & 0,054 & 0,312 & 0,001 & 0,143 & 0,248 & 0,721 & 0,002 \\
\hline
\end{tabular}

a,b,c Medias de tratamiento o efecto principal con distinta literal indica diferencias estadísticas $(\mathrm{P} \leq 0,005)$. EE=Error estándar

$\mathrm{T}=$ Tratamiento; $\mathrm{PC}=$ Proteína cruda $(\%) ; \mathrm{EM}=$ Energía metabolizable $\left(\mathrm{Mcal} \mathrm{kg}^{-1}\right) ; \mathrm{GDP}=$ Ganancia diaria de peso $\left(\mathrm{kg} \mathrm{d}^{-1}\right) ; \mathrm{CDA}=\mathrm{Consumo}$ diario de alimento $\left(\mathrm{kg} \mathrm{d}^{-1}\right) ; \mathrm{CA}=$ Conversión alimenticia; $\mathrm{PI}=$ Peso inicial $(\mathrm{kg}) ; \mathrm{PF}=$ Peso final $(\mathrm{kg}) ; \mathrm{GCM}=$ Ganancia diaria de carne magra $\left(\mathrm{kg} \mathrm{d}^{-1}\right) ; \mathrm{GD}=\mathrm{Grasa}$ dorsal (mm); $\mathrm{AML}=$ Área de músculo longissimus $\left(\mathrm{cm}^{2}\right) ; \mathrm{PCM}=$ Carne magra $(\%)$; $\mathrm{U}=$ Concentración de urea en plasma $\left(\mathrm{mg} \mathrm{dL}^{-1}\right)$.

\section{AGRADECIMIENTOS}

Se agradece al Consejo Nacional de Ciencia y Tecnología (CONACYT) por la beca otorgada al primer autor. Esta investigación fue parcialmente financiada por el Fideicomiso Revocable de Administración e Inversión No. 167304-2008 del Colegio de Postgraduados.

\section{REFERENCIAS}

Chaney AL, EP Marbach. 1962. Modified reagents for determination of urea and ammonia. Clin Chem 8, 130-132.

Deng D, K Yao, W Chu, T Li, R Huang, Y Yin, G Wu. 2009. Impaired translation initiation activation and reduced protein synthesis in weaned piglets fed a low-protein diet. J Nutr Biochem 20, 544-552.

Feilberg A, L Dezhao, PSA Anders, JH Michael, ENJ Kristoffer. 2010. Odorant emissions from intensive pig production measured by online proton-transfer-reaction mass spectrometry. Enviro Sci Technol 44, 5894-5900.

Figueroa JL, AJ Lewis, PS Miller, RL Fischer, RS Gómez, RM Diedrichsen. 2002. Nitrogen metabolism and growth performance of gilts fed standard corn-soybean meal diets or low-crude protein, amino acid-supplemented diets. J Anim Sci 80, 2911-2919.

Figueroa JL, AJ Lewis, PS Miller, RL Fischer, RM Diedrichsen. 2003. Growth, carcass traits, and plasma amino acid concentrations of gilts fed low-protein diets supplemented with amino acids including histidine, isoleucine, and valine. J Anim Sci 81, 1529-1537.

Figueroa JL, M Cervantes, M Cuca, M Méndez. 2004. Growth performance of growing-finishing pigs fed diets low in protein and energy. Agrociencia 38, 383-394.

Figueroa JL, J Estrada, V Zamora, JL Cordero, MT Sánchez-Torres, R Nieto, JMF Copado. 2012. Digestible lysine levels in low-protein diets supplemented with synthetic amino acids for nursery, growing, and finishing barrows. Irish J Agric Food Res 51, 33-44.

Gómez RS, AJ Lewis, PS Miller, HY Chen. 2002. Growth performance, diet apparent digestibility and plasma metabolite of barrows fed corn-soybean meal diets or low-protein, amino acid supplemented diets at different feeding levels. J Anim Sci 80, 644-653.

Hayes ET, ABG Leek, TP Curran, VA Dodd, OT Carton, VE Beattie, JV O'Dohert. 2004. The influence of dietary crude protein level on odor and ammonia emissions from finishing pig houses. Biores Technol 91, 309-315.

Heger J, S Mengesha, D Vodehnal. 1998. Effect of essential: total nitrogen ratio on protein utilization in the growing pig. Br J Nutr 80, 537-544.

Kerr BJ, LL Southern, TD Bidner, KG Friesen, RA Easter. 2003. Influence of dietary protein level, amino acid supplementation, and dietary energy levels on growing-finishing pig performance and carcass composition. J Anim Sci 81, 3075-3087.

Le Bellego L, J Van Milgen, S Dubois, J Noblet. 2001. Energy utilization of low-protein diets in growing pigs. J Anim Sci 79, 1259-1271.

Le Bellego L, J Van Milgen, J Noblet. 2002. Effect of high temperature and low-protein diets on the performance of growing-finishing pigs. J Anim Sci 80, 691-701.

Martínez-Aispuro M, JL Figueroa-Velasco, JE Trujillo-Coutiño, V Zamora-Zamora, JL Cordero-Mora, MT Sánchez-Torres, L ReynaSantamaría. 2009. Respuesta productiva y concentración de urea en plasma de cerdos en crecimiento alimentados con dietas sorgo-pasta de soya con baja proteína. Vet Méx 40, 27-38.

Martínez-Aispuro M, JL Figueroa-Velasco, V Zamora-Zamora, MT Sánchez-Torres, ME Ortega-Cerrilla, JL Cordero-Mora, A Ruiz-Flores, S González-Muñoz. 2012. Effect of fatty acids source on growth performance, carcass characteristics, plasma urea nitrogen concentration, and fatty acid profile in meat of pigs fed standard- or low-protein diets. Spanish J Agric Res 10, 993-1004.

National Pork Producers Council. 1991. Procedures to evaluate market hogs. $3^{\text {rd }}$ ed. National Pork Producers Council. Des Moines, IA, USA.

NRC (National Research Council), 1998. Nutrient Requirements of Swine. $10^{\text {th }}$ ed. National Academy Press, Washington, D.C., USA.

Nyachoti CM, FO Omogbenigun, M Rademacher, G Blank. 2006. Performance responses and indicators of gastrointestinal health in early-weaned pigs fed low-protein amino acid-supplemented diets. J Anim Sci 84, 125-134.

Osada T, R Takada, I Shinzato. 2011. Potential reduction of greenhouse gas emission from swine manure by using a low-protein diet supplemented with synthetic amino acids. Anim Feed Sci Technol 166, 562-574.

Reyes I, JL Figueroa, MA Cobos, MT Sánchez-Torres, V Zamora, JL Cordero. 2012. Probiótico (Enterococcus faecium) adicionado a dietas estándar y con baja proteína para cerdos. Arch Zootec 61, 589-598.

Reyna L, JL Figueroa, V Zamora, JL Cordero, MT Sánchez-Torres, M Cuca. 2006. Addition of protease to standard diet or low protein, amino acid-supplemented, sorghum- soybean meal diets for growingfinishing pigs. J Anim Vet Adv 12, 1202-1208.

Rivera A, JL Figueroa, E Saldaña, V Zamora, MT Sánchez-Torres, JL Cordero. 2010. Finalización de cerdos con baja proteína y mananooligosacáridos o nucleótidos. Arch Zootec 3, 357-368.

SAS Institute Inc SAS/STAT User's Guide, Second Edition, 2009. SAS Institute Inc. Cary, NC. USA.

Shriver JA, SD Carter, AL Sutton, BT Richert, BW Senne, LA Pettey. 2003. Effects of adding fiber sources to reduced-crude protein, amino acid-supplemented diets on nitrogen excretion, growth performance, and carcass traits of finishing pigs. J Anim Sci 81, 492-502. 
Tuitoek K, LG Young, CFM de Lange, BJ Kerr. 1997. The effect of reducing excess dietary amino acids on growing-finishing pig performance: an evaluation of the ideal protein concept. $J$ Anim Sci $75,1575-1583$.

Ward TL, LL Southern. 1995. Sorghum amino acid-supplemented diets for the 50 to 100 kilogram pig. J Anim Sci 73, 1746-1753.

Yue LY, SY Qiao. 2008. Effects of low-protein diets supplemented with crystalline amino acids on performance and intestinal development in piglets over the first 2 weeks after weaning. Livest Sci $115,144-152$.

Zamora V, JL Figueroa, JL Cordero, M Rugerio, L Reyna, MT SánchezTorres. 2010. Addition of glucomannans to low-protein diets based on sorghum-soybean meal for growing and finishing pigs. Revista Científica FCV-LUZ 20, 274-283.

Zervas S, RT Zijlstra. 2002. Effects of dietary protein and oat hull fiber on nitrogen excretion patterns and postprandial plasma urea profiles in grower pigs. J Anim Sci 80, 3238-3246. 
